\title{
Vaccine Adjuvant
}

National Cancer Institute

\section{Source}

National Cancer Institute. Vaccine Adjuvant. NCI Thesaurus. Code C2554.

Substances used in vaccines to enhance the ability of antigens to elicit an immune response. 